\title{
Towards accurate estimation of fast varying frequency in future electricity networks: The transition from model- free methods to model-based approach
}

Proc IMechE Part I:

J Systems and Control Engineering 2016, Vol. 230(10) II64-1175 (c) IMechE 2016

Reprints and permissions:

sagepub.co.uk/journalsPermissions.nav DOI: I0.1।77/095965|8|6670040

pii.sagepub.com

@SAGE

\author{
Alexander Stotsky
}

\begin{abstract}
Accurate estimation of fast varying fundamental frequency in the presence of harmonics and noise will be required for effective frequency regulation in future electricity networks with high penetration level of renewable energy sources. Two new algorithms for network frequency tracking are proposed. The first algorithm represents a robust modification of classical zero crossing method, which is widely used in industry. The second algorithm is a multiple model algorithm based on the systems with harmonic regressor. Algorithm allows complete reconstruction of the frequency content of the signal, using information about the upper bound of the number of harmonics only. Moreover, new family of highorder algorithms together with new stepwise splitting method are proposed for parameter calculation in systems with harmonic regressor for the accuracy improvement. Statistical methods are introduced for comparison of two new algorithms to classical zero crossing algorithm. The modified algorithm provides significant improvement compared to the classical algorithm, and the algorithm with harmonic regressor provides further improvement of the statistical performance indexes with respect to the modified algorithm.
\end{abstract}

\section{Keywords}

Accurate frequency tracking in future electricity networks, harmonic regressor, multiple model, statistical quantification methods of comparison of algorithms, high-order algorithms, stepwise splitting, inversion of ill-conditioned matrices

Date received: 2 March 2016; accepted: 19 August 2016

\section{Introduction}

High penetration level of (1) renewable energy sources, (2) power electronics, (3) advanced transmission systems (like high-voltage direct current (HVDC) and others) and (4) higher nonlinear loads and new types of loads in future electricity networks will (1) essentially reduce grid inertia, (2) induce faster variations of grid frequency in the case of load events and (3) introduce significant distortions (e.g. additional harmonics) in voltage and current signals.

Fast deviations from fundamental frequency, appearance of additional harmonics and noise will result in reduction in efficiency of equipment, power losses, heating, increased noise levels and others.

Frequency regulation, enabled via active power control of wind turbines, load-side control and others will play an important role in future electricity networks for performance improvement. Notice that reliable frequency measurement is necessary for high-performance network control as well as for system protection. Errors in frequency estimates will result in erroneous control action and even in frequency oscillations. This article addresses a very important issue of accuracy improvement of frequency estimation algorithms in the presence of harmonics and noise (electrical noise, measurement noise and others) in future electricity networks.

A brief overview of existing frequency estimation methods is given below. All the methods are classified into two large groups: model-free methods and

Division of Electric Power Engineering, Department of Energy and Environment, Chalmers University of Technology, Gothenburg, Sweden

\section{Corresponding author:}

Alexander Stotsky, Division of Electric Power Engineering, Department of Energy and Environment, Chalmers University of Technology, SE-4I2 96 Gothenburg, Sweden.

Email: alexander.stotsky@telia.com; alexander.stotsky@chalmers.se 
model-based methods. Recent modifications of zero crossing method as a model-free method, which is widely used in industrial applications, are listed in section "Frequency estimation with zero crossing method as a model-free approach." Recent advances in modelbased methods are briefly summarized in section "Existing model-based frequency estimation methods and further improvement of the accuracy." The contributions of this article are also outlined in section "Frequency estimation with zero crossing method as a model-free approach" with respect to the previous work and summarized in section "Existing model-based frequency estimation methods and further improvement of the accuracy."

\section{Frequency estimation with zero crossing method as a model-free approach}

Zero crossing detection and calculation of the number of cycles that occur in a predetermined time interval is a direct, simple and widely used methodology for frequency detection. A zero crossing is a point where the sign of a signal changes. The signal is approximated by linear function between two adjacent samples around the zero crossing point for accurate detection of the point. ${ }^{1}$ The frequency of the signal is estimated by detection of the times when the signal crosses zero. The time between each crossing is equal to the half of the period of the signal. The method is a model-free approach (the method of direct measurement), and it is widely used in many applications (mostly in electrical engineering applications) due to simplicity and robustness with respect to disturbances which do not occur around zero crossing point. However, the performance of the method is significantly affected by the measurement noise and higher harmonics. In particular, multiple zero crossing events (which are close to each other) appear due to measurement noise and higher harmonics.

A number of modifications, which improve zero crossing method for frequency detection in different applications, are reported in the literature ${ }^{2-6}$ (see references therein). These methods suffer more or less from the following drawbacks: (1) slow response and inaccuracies in the case of frequency tracking, (2) applicability to certain types of signals and/or in postprocessing only and (3) computational complexity and some others.

A simple and easy-to-implement modification of zero crossing method with minimal delay, which is applicable in real time for any type of signals, is described in section "Modification of zero crossing method: improvement of estimation accuracy." The algorithm overcomes the major part of the drawbacks listed above. Statistical performance quantification methods show significant improvement of the frequency detection and tracking accuracy compared to classical algorithm, see section "Simulation results and comparisons with classical method." Limitations of the method are described in section "Limitations of the method." Further improvements can be made using model-based estimation methods, introduced in section "Existing model-based frequency estimation methods and further improvement of the accuracy."

\section{Existing model-based frequency estimation methods and further improvement of the accuracy}

A number of interesting surveys on model-based frequency estimation is available in the literature ${ }^{7-9}$ (see references therein). A brief overview of existing frequency estimation methods with application to electricity networks is given below.

In general, frequency estimators can be classified as single and multi-frequency estimators designed in continuous and discrete time domains. Multi-frequency estimation can be multi-harmonic frequency estimation, where each component of the signal is harmonically related to fundamental frequency, and multi-tone frequency estimation, where the signal contains multiple frequency components, which are not related to each other. Multi-harmonic frequency estimation is usually applied in electricity networks, where each component of the signal is harmonically related to the fundamental frequency of network.

Recent developments in continuous time frequency estimation are reported in previous studies ${ }^{10-12}$ for single frequency case and in $\mathrm{Xia}^{13}$ for multi-frequency case (see also references therein). Notice that errors related to discretization of continuous time algorithms together with difficulties associated with quantification of the performance of discretized system in the presence of different types of errors give the first preference to discrete time algorithm design in digital signal processing applications. Continuous time algorithms are more suitable for estimation of periodic disturbances for control of continuous time systems. ${ }^{12}$ In addition, discrete time design covers larger class of algorithms since many discrete time algorithms do not have continuous time counterparts. Finally, accurate estimation of multifrequency signals is not possible with single frequency model.

Multi-frequency discrete time estimator, where the model of multi-frequency signal is presented in the form of linear difference equation with unknown coefficients, which define the frequencies and amplitudes, can be found, for example, in Stotsky ${ }^{14}$ (see also references therein). The method which was designed for frequency detection may be inaccurate in the case of frequency tracking. Besides, this method and similar methods, see, for example, Quinn and Hannan, ${ }^{9}$ are sensitive to unmodeled harmonics, measurement noise, numerical inaccuracies and others. 
Promising multi-harmonic frequency estimators are based on optimization techniques, which maximize periodogram as a function of frequency, ${ }^{15}$ or minimize the error sum of squares with respect to unknown quantities, such as frequencies, phase shifts and coefficients. The latter, which is often called nonlinear least-squares method, is also associated with the maximumlikelihood approach. ${ }^{16}$ The best model matching provides the most accurate estimates. However, a number of extremum seeking algorithms, which are often realized as iterative search procedures, can be ineffective due to local extrema and restricted region of attraction, which in turn are present due to a highly nonlinear nature of the problem. Computational complexity is an additional problem associated with the search procedures.

These difficulties can be avoided by applying multiple model approach, where the set of models is defined and each model is associated with different fundamental frequency. Residual error, which is associated with this set, can be presented as a function of frequency and the frequency which corresponds to the minimal value of residual error is the true frequency. Moreover, minimal residual error is also associated with the variance of the measurement noise. ${ }^{17}$ Accuracy of calculation of residual errors plays an important role in the frequency determination, using this method. Each model in the set represents the system with harmonic regressor, and the parameters of the models can be calculated using recently developed high-order algorithms, ${ }^{18,19}$ which provide high accuracy of the parameter and residual error estimation. The family of high-order algorithms is extended in Appendix 1, where new stepwise splitting method is also developed.

All the residual errors can be calculated simultaneously, using parallel calculations, which essentially reduce execution time of the algorithm.

A simple and computationally efficient minimum seeking algorithm, realized as the interval reduction method, is developed in section "Systems with harmonic regressor: multiple model approach" for fast and accurate calculation of the minimal value of the residual error and high-performance estimation of the frequency and the variance of the measurement noise.

Statistical performance quantification methods show significant improvement of the frequency tracking accuracy of model-based algorithm described in section "Systems with harmonic regressor: multiple model approach" compared to classical model-free zero crossing algorithm and modified algorithm (described in section "Modification of zero crossing method: improvement of estimation accuracy"), see comparisons in section "Comparison of the frequency tracking algorithms" and discussion in section "Conclusion and discussion: the transition from zero crossing method to complete reconstruction of the frequency contents of the signals."
Finally, the contributions of this article can be summarized as follows:

1. New simple and easy-to-implement modification of zero crossing method with minimal delay, which is applicable in real time for any type of signals;

2. New multi-harmonic frequency estimator based on multiple model approach with simple and computationally efficient minimum seeking algorithm for residual error, realized as the interval reduction method;

3. New family of high-order algorithms and new stepwise splitting method for accurate calculations of the inverse of information matrix.

\section{Modification of zero crossing method: improvement of estimation accuracy}

\section{Description of the method}

Accuracy improvement in detection of location of zeros using information around two adjacent samples around the zero crossing point is the main idea for robust modification of classical zero crossing method. A local coordinate system in the form of the window is defined as soon as a zero is detected using conventional method (see Figure 1). The accuracy of zero detection is improved after several steps defined by the window size mentioned above. Linear model of the signal is fitted to the measured data in the least-squares sense in the local coordinate system. Zero is calculated with linear interpolation in the system of local coordinates and transformed to the global coordinate system. Notice that the window is usually asymmetric with respect to zero crossing point. Few samples only are used after zero crossing point, minimizing a delay in frequency estimation for control. Figure 1 shows that this method can be successfully applied for lumping together two zeros which are close to each other.

Notice that double/multiple zeros which are close to each other is a significant problem in the classical zero crossing frequency detection algorithm. The time period calculated for these two neighboring zeros is very short, and the estimated frequency which is very high is usually rejected as an outlier. The methods, which pick up one of these zeros rejecting other ones, have low estimation accuracy, and the histogram method for averaging multiple zeros ${ }^{4}$ is effectively applicable for a certain type of signals only. The method proposed in this article is efficient for lumping together multiple zeros which are close to each other and significantly improves accuracy of detection of zeros in the presence of any disturbances around zero crossing point (see Figure 1(b)). The method has one parameter only to be adjusted, this being a size of the window. The method is applicable for a wide class of signals and the performance can be restrictedly adjusted via the choice of the window size. 


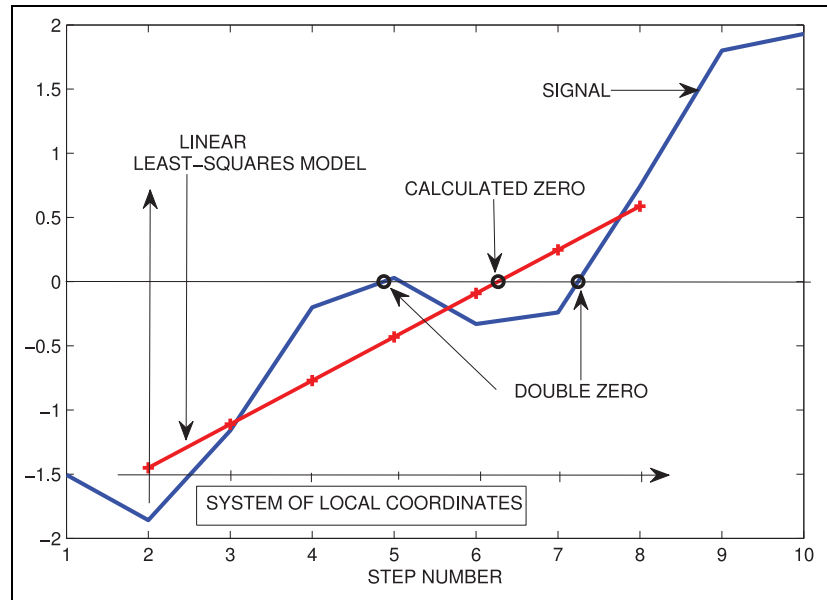

(a)

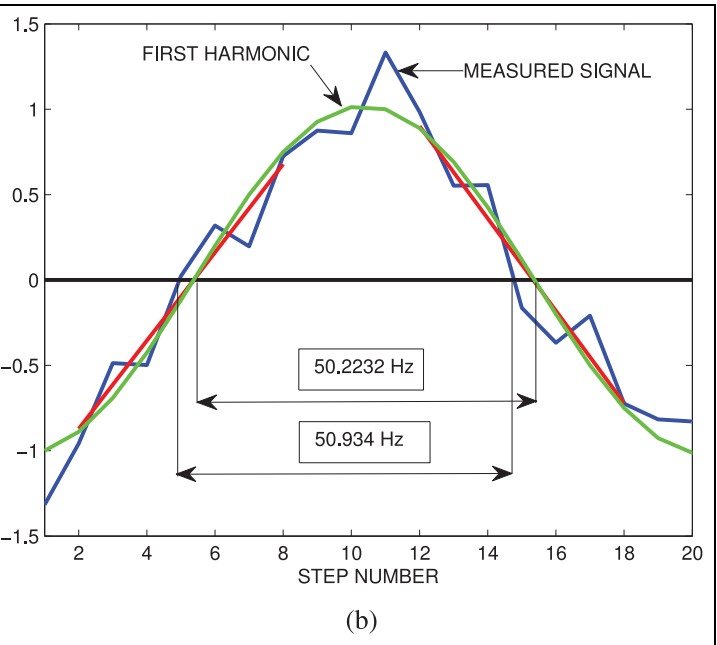

(b)

Figure I. A signal with the frequency of $50 \mathrm{~Hz}$ with the third and the seventh harmonic contaminated with a white noise is plotted in subplot (a) aiming to show double zero avoidance with improved zero crossing detection algorithm. The signal for which zero crossing should be detected is plotted with a blue line. The system of local coordinates in the form of the window is defined as soon as a zero (plotted with a round sign of a black color) is detected using conventional method of linear interpolation between two adjacent samples around the zero crossing point. Linear model of the signal (plotted with a red line) is fitted to the measured data in the least-squares sense in the local coordinate system, and zero of linear function (plotted with a round sign of a black color) is calculated using linear interpolation. A signal with the frequency of $50 \mathrm{~Hz}$ with third and seventh harmonics contaminated with a white noise is plotted with a blue line in subplot (b). The first harmonic is plotted with a green line. A linear model of the signal (plotted with a red line) is fitted to the measured data plotted with a blue line around zero points in the least-squares sense. The frequency determined by classical zero crossing algorithm is $50.934 \mathrm{~Hz}$ and the frequency determined by modified algorithm is $50.2232 \mathrm{~Hz}$. Modification of zero crossing algorithm described in section "Modification of zero crossing method: improvement of estimation accuracy" essentially improves estimation accuracy in the presence of noise and harmonics.

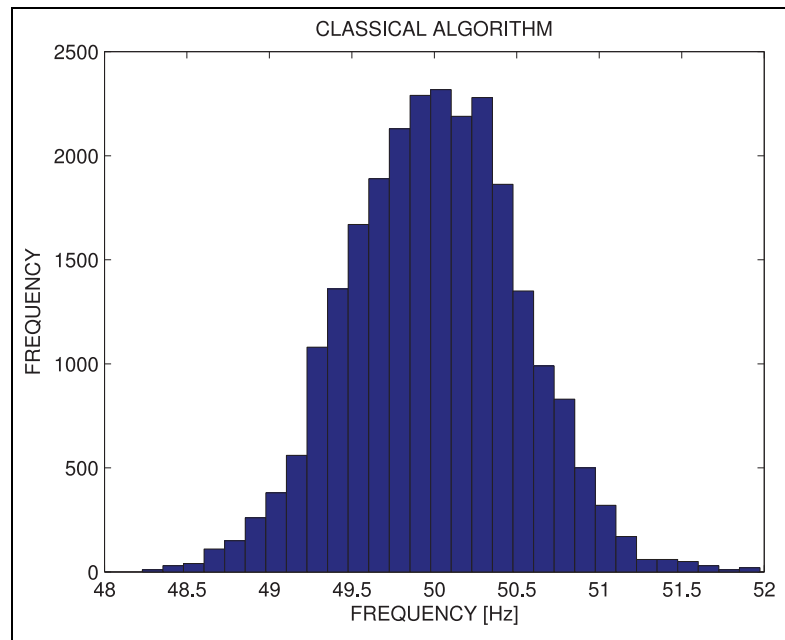

(a)

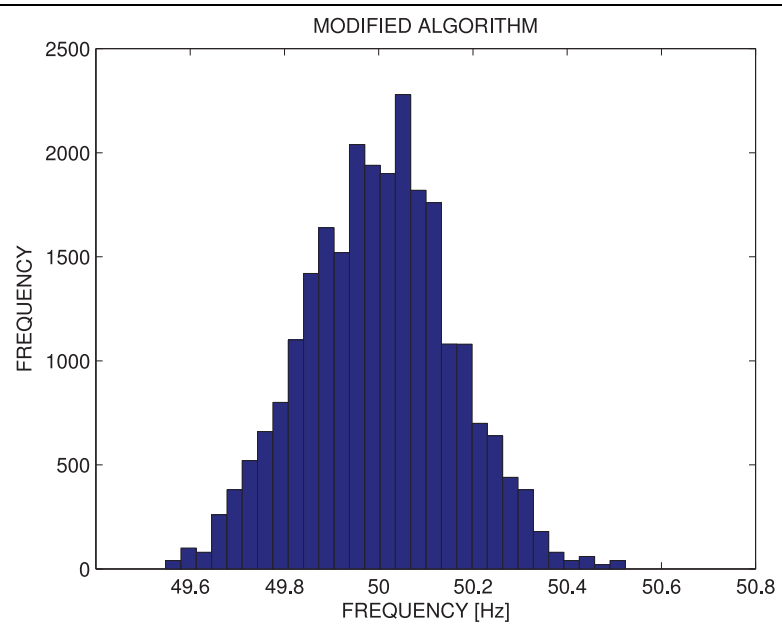

(b)

Figure 2. A signal with the frequency of $50 \mathrm{~Hz}$ with the third and the seventh harmonic was contaminated with a white noise and processed by classical and modified zero crossing algorithms. The histogram of frequency estimation with classical zero crossing algorithm is plotted in subplot (a). The histogram of frequency estimation with modified zero crossing algorithm is plotted in subplot (b).

\section{Simulation results and comparisons with classical method}

The performance of modified algorithm is quantified in Figure 2, where two histograms, which evaluate estimation accuracy of the system frequency, are presented. A signal with the frequency of $50 \mathrm{~Hz}$ with the third and the seventh harmonic was contaminated with a white noise and processed by classical and modified zero crossing algorithms. Statistical methods are applied for comparison and quantification of the performance of classical and modified zero crossing algorithms. Statistical methods are associated with one-sample $t$ and two-sample $F$ hypothesis tests. One-sample $t$-test shows that both algorithms estimate actual frequency 
of $50 \mathrm{~Hz}$ statistically correct. The test indicates a failure to reject the null hypothesis that the mean value of both data sets is $50 \mathrm{~Hz}$ at a sufficiently low significance level. Two-sample $F$-test for equal variances shows that the reduction of the variance provided by the modified algorithm is statistically significant. It means that modified algorithm provides much better accuracy of the frequency detection.

\section{Limitations of the method}

A time delay associated with the curve fitting is the first drawback of this method. Besides, the accuracy of estimation deteriorates (due to linearity of the model around zero crossing point, see Figure 1) with more significant influence of harmonics. The model-based frequency estimation method, described in the next section, overcomes these difficulties.

\section{Systems with harmonic regressor: multiple model approach}

\section{Description of the minimal residual method}

Suppose that a measured signal $y_{k}$ can be presented in the following form

$$
y_{k}=\varphi_{k}^{T} \theta_{*}+\xi_{k}
$$

where $\theta_{*}$ is the vector of unknown constant parameters and $\varphi_{k}$ is unknown harmonic regressor presented in the following form

$$
\begin{aligned}
& \varphi_{k}^{T}=\left[\cos \left(q_{0} k\right) \sin \left(q_{0} k\right) \cos \left(2 q_{0} k\right)\right. \\
& \left.\sin \left(2 q_{0} k\right) \ldots \cos \left(h q_{0} k\right) \sin \left(h q_{0} k\right)\right]
\end{aligned}
$$

where $q_{0}$ is unknown fundamental frequency of network (e.g. $q_{0}=50 \mathrm{~Hz}$ or $q_{0}=60 \mathrm{~Hz}$ ), $h$ is unknown number of harmonics and $\xi_{k}$ is a zero mean white Gaussian noise, $k=1,2, \ldots$ is the step number. The system has three following unknown quantities: the fundamental frequency of network $q_{0}$, the number of harmonics $h$ and the vector of parameters $\theta_{*}$. It is assumed that the upper bound $\bar{h}$ of the number of harmonics is known and $h \leqslant \bar{h}$. The algorithm for frequency and parameter estimation can be presented in the following steps, which are executed in each step $k$ :

Step 1: Multiple model estimation for initial set of frequencies. Define the frequency interval as the following vector of size $r$

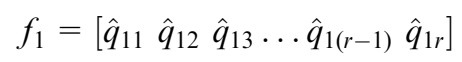

where the frequencies $\hat{q}_{1 i}, i=1, \ldots, r, r \geqslant 3$ are presented in increasing order. The frequency interval should cover unknown fundamental frequency of the system $q_{0}$.
Substep 1: Multiple model estimation of the variance. The regressor vector $\hat{\varphi}_{i}$ is introduced for each frequency $\hat{q}_{1 i}$ as follows

$$
\begin{aligned}
& \hat{\varphi}_{i}^{T}=\left[\cos \left(\hat{q}_{1 i} k\right) \sin \left(\hat{q}_{1 i} k\right) \cos \left(2 \hat{q}_{1 i} k\right)\right. \\
& \left.\sin \left(2 \hat{q}_{1 i} k\right) \ldots \cos \left(\bar{h} \hat{q}_{1 i} k\right) \sin \left(\bar{h} \hat{q}_{1 i} k\right)\right]
\end{aligned}
$$

forming a multiple model of the regressor with the frequencies corresponding to the components of the vector (3). Notice that the size of the model of each regressor (4) is larger than or equal to the size of unknown regressor (2) since $h \leqslant \bar{h}$.

Multiple model of the signal (1) with adjustable parameters $\theta_{i}$ is presented in the following form

$$
\hat{y}_{i}=\hat{\varphi}_{i}^{T} \theta_{i}
$$

The signal $y_{k}$ is approximated by the multiple model $\hat{y}_{i}$ for each frequency corresponding to the components of the vector (3) in the least-squares sense in each step $k$ of a moving window of a size $w$.

The frequency estimation algorithm is based on minimization of the following error $E_{i}$ with respect to argument $i$, which corresponds to the certain frequency in the multiple model (5)

$$
E_{i}=\sum_{p=k-(w-1)}^{p=k}\left(\hat{y}_{p i}-y_{p}\right)^{2}
$$

for a fixed step $k$, where $k \geqslant w$.

The least-squares solution for estimation of the parameter vector $\theta_{i}$ can be written as follows

$$
\begin{aligned}
& A_{i} \theta_{i}=b_{i} \\
& A_{i}=\sum_{p=k-(w-1)}^{p=k} \hat{\varphi}_{p i} \hat{\varphi}_{p i}^{T} \\
& b_{i}=\sum_{p=k-(w-1)}^{p=k} \hat{\varphi}_{p i} y_{p}
\end{aligned}
$$

where the matrix $A_{i}$ is an information matrix, and the parameter vector $\theta_{i}$ satisfies (7). The parameter vector is calculated with high accuracy using high-order algorithms, described in Stotsky, ${ }^{18,19}$ see also Appendix 1 and section "Accuracy, high-order algorithms, stepwise splitting and parallel computing" for details.

Finally, the variance $V_{i}$ of the measurement noise $\xi_{k}$, associated with the multiple model is defined as follows

$$
V_{i}=\frac{E_{i}}{w-2 \bar{h}-1}
$$

Substep 2: Calculation of the frequency via minimization of the variance. The model for $V_{i}$ is defined as follows

$$
\hat{V}=a q^{2}+b q+c
$$




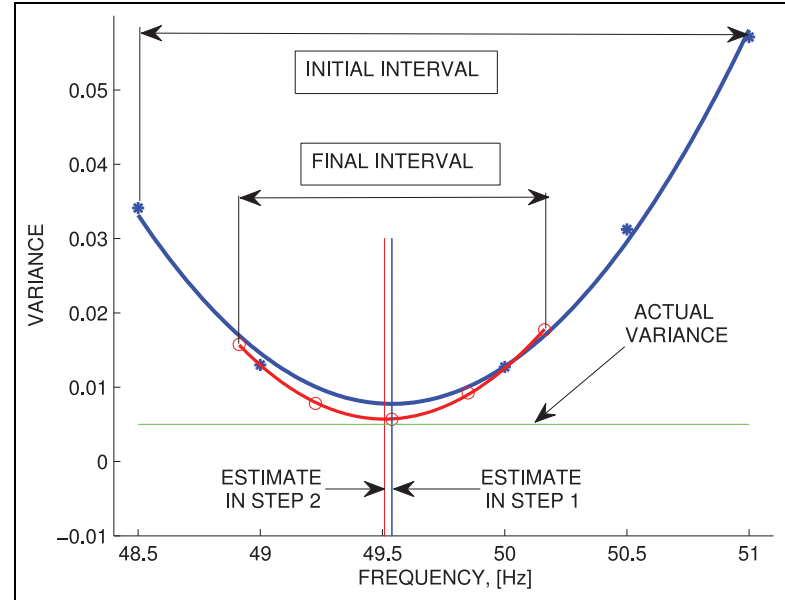

Figure 3. Estimation of the frequency in two steps. The following set of frequencies is selected in the first step of estimation [48.5 $495050.55 \mathrm{I}] \mathrm{Hz}$. This set covers actual frequency of $49.5 \mathrm{~Hz}$. Each point in this set is plotted with a star sign of a blue color. The frequency of $49.5382 \mathrm{~Hz}$ was determined after the first step of estimation as an argument corresponding to the minimal value of parabola plotted with a blue line. The frequency of $49.5382 \mathrm{~Hz}$ is used as the central point of new set of frequencies with the range, which is reduced compared to the range of the initial set. The following set of frequencies was determined in the second step of estimation [48.9I32 4949.2257 49.538249.8507 5050.1632] Hz, where the frequencies 49 and $50 \mathrm{~Hz}$ were transferred from the initial interval. Each point in this set (excepting the frequencies of 49 and $50 \mathrm{~Hz}$ ) is plotted with a round sign of a red color. The frequency of $49.5079 \mathrm{~Hz}$ was determined in the second step of estimation in the same way. Minimal value of parabola plotted with a red line corresponds to the actual value of the variance of measurement noise, which is equal to 0.005 .

where $q$ is the frequency and $a, b$ and $c$ are the coefficients calculated using least-squares method to provide the best fit of $V_{i}$ over the frequency interval $f_{1}$. Estimated frequency is defined as

$$
\bar{q}_{1}=-\frac{b}{2 a}
$$

which corresponds to the minimal value of $\hat{V}$, which is also an estimate of the variance of measurement noise $\xi_{k}$.

The frequency $\bar{q}_{1}$ is used as the central point for interval, which should be chosen in the next step.

Notice that the initial frequency interval (3) should be sufficiently large in order to cover unknown frequency. However, inaccuracies in calculations of the variances $V_{i}$ for each model, especially in the points located close to the boundaries, result in a biased estimate of the frequency. These inaccuracies have more significant impact on larger intervals and therefore the frequency interval should be reduced in the next step of estimation for the sake of accuracy improvement.

Step 2: Multiple model estimation for updated set of frequencies. Define updated frequency interval as the following vector of size $r$

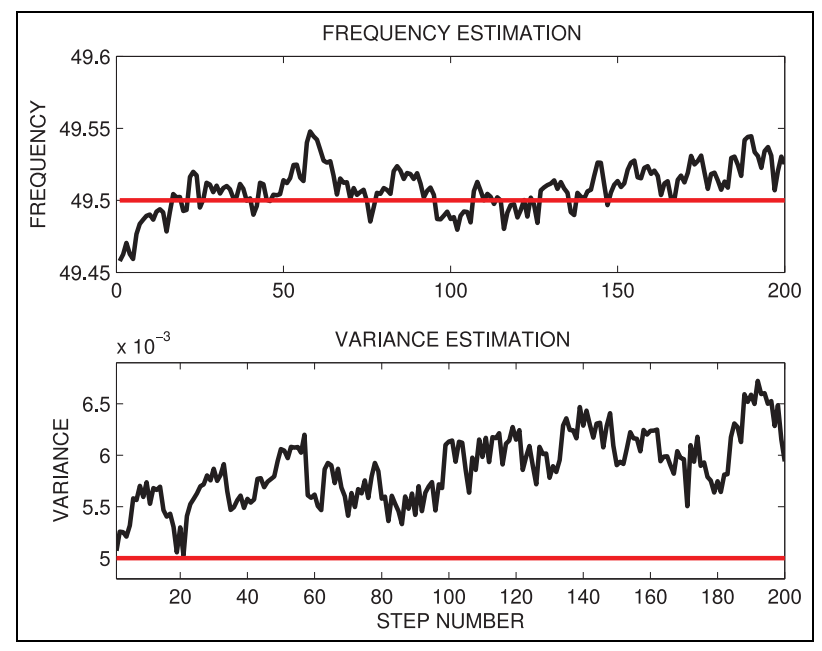

Figure 4. A time chart of frequency estimation is presented in the first subplot, where the frequency signal is plotted with a black line and actual frequency, which is equal to $49.5 \mathrm{~Hz}$, is plotted with a red line. The second subplot is a time chart of estimation of the variance of measurement noise, which is plotted with a black line, and actual variance, which is equal to 0.005 , is plotted with a red line.

$$
f_{2}=\left[\begin{array}{lllll}
\hat{q}_{21} & \hat{q}_{22} \ldots \bar{q}_{1} \ldots \hat{q}_{2(r-1)} & \hat{q}_{2 r}
\end{array}\right]
$$

where the range of the frequency interval defined by $f_{2}$ in (13) is reduced with respect to the range of the frequency interval defined by $f_{1}$ in (3), that is, $\left(\hat{q}_{2 r}-\hat{q}_{21}\right)<<<\left(\hat{q}_{1 r}-\hat{q}_{11}\right)$.

Notice that the frequencies from the interval $f_{1}$ can be included in the interval $f_{2}$ (if they fit to this interval), which improves curve fitting accuracy without additional computational effort. The substeps 1 and 2 are repeated for this new set of frequencies (13) resulting in updated estimate of the frequency $\bar{q}_{2}$, which is used as central point for interval defined in the next step.

Reduction of the range of the interval in each step ensures the convergence of estimated frequency $\bar{q}_{z}$, where $z=1,2, \ldots$ to its true value $q_{0}$ as the step number increases and the range of the interval reaches its minimal value.

This algorithm is the fast convergent algorithm due to the model-based minimization of the variance in each step and few steps are required only for estimation of the fundamental frequency with a very high accuracy. Notice that two steps are usually sufficient for accurate estimation of the frequency.

The algorithm of minimization of the variance and estimation of the frequency in two steps is illustrated in Figure 3. A time chart of the frequency and variance estimation for the same case is plotted in Figure 4. Finally, estimation of the first harmonic of the signal is plotted in Figure 5.

Notice that any overtones, including half harmonics, can be included in the model (4) provided that these overtones are present in the signal (1). Inclusion of 


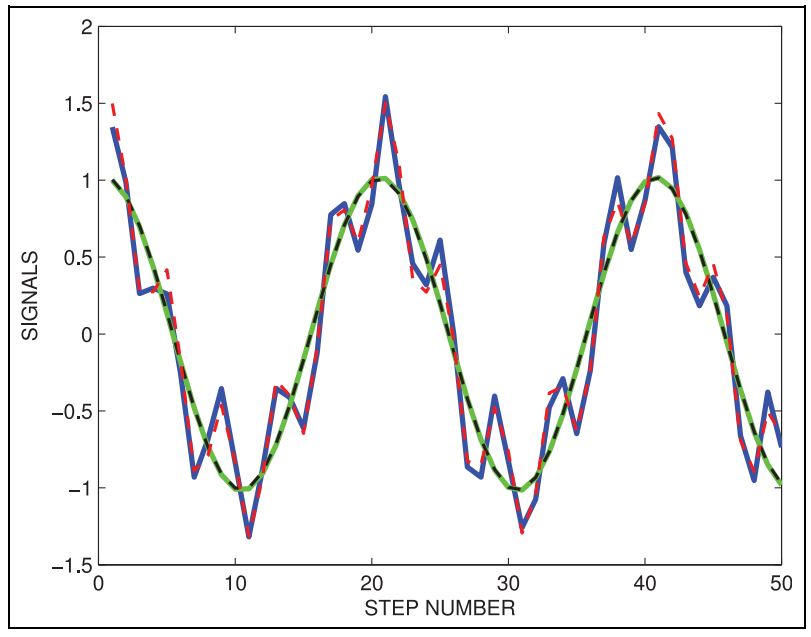

Figure 5. Estimation of the first harmonic of the signal. The signal with the frequency of $49.5 \mathrm{~Hz}$ with the second and the fifth harmonic was contaminated with a white measurement noise. The signal is plotted with a blue solid line, and its first harmonic is plotted with green solid line. Approximations of the signal and the first harmonic are plotted with red and black dashed lines, respectively.

additional number of overtones increases the size of the regressor vector. This size together with the window size $w$ can be reduced using the stepwise regression method of subsequent inclusion of the harmonics/overtones in the regressor. ${ }^{17}$ This increases computational complexity of the algorithm, but provides more accurate estimate in the case of fast frequency transients.

Notice also that a priori (inaccurate) estimate of the frequency obtained with other methods such as zero crossing method, the method of estimating coefficients of difference equations ${ }^{9,14}$ and others can be used as a starting point for choosing initial frequency interval in Step 1. Accuracy of the frequency estimate can be improved further, using the method described in this section. Computational complexity of the algorithm can be reduced, since a minimal length of the interval can be used only (and the algorithm converges in one step in this case), provided that a priori estimate is accurate enough.

\section{Accuracy, high-order algorithms, stepwise splitting and parallel computing}

Accuracy of the frequency estimation depends on the curve fitting accuracy, which in turn depends on the number of frequencies (the number of models in the set) and the accuracy of estimation of the variance $V_{i}$ for each harmonic regressor. The latter in turn depends on the accuracy of calculation of the parameter vector $\theta_{i}$ for each regressor. This indicates a trade-off between the frequency estimation accuracy and computational complexity and hence the accuracy can be improved with additional computational burden.
The parameter vector can be calculated with high accuracy, using high-order algorithms, described in Stotsky ${ }^{18}$ for an SDD (strictly diagonally dominant) information matrix $A_{i}$ (for a sufficiently large window size), and for a positive definite information matrix (for a small window size), described in Stotsky. ${ }^{19}$ A large window size is used in the case of relatively slow variations of the frequency, and fast frequency variations can be accurately estimated in the window of a small size.

A new family of high-order algorithms is described in Appendix 1, where new matrix splitting methods are also proposed. Performance of new high-order algorithms is compared to the performance of existing algorithms. A new computationally efficient method, named as stepwise splitting for accurate recursive calculation of the inverse of information matrix (which is a positive definite matrix for a small window size) in moving window, is also described there. Stepwise splitting method is very efficient even for inversion of illconditioned and rank-deficient matrices.

Parallel computing associated with multiple processing units (such as a single computer with multiple processors, several networked computers, specialized hardware and others) can be applied for reduction of computational complexity via simultaneous execution of high-order algorithms for estimation of parameters and variance for each harmonic regressor in multiple model, where the number of models has a direct impact on estimation accuracy. Each processing element should calculate the variance for each harmonic regressor simultaneously, and the results of these calculations are applied further for minimization of the variance.

Parallel computing provides computational enhancement and essentially reduces execution time of the algorithm, ${ }^{20}$ which allow accurate estimation of the variance for each harmonic regressor and hence accurate estimation of the frequency.

\section{Comparison of the frequency tracking algorithms}

Comparison of classical and modified zero crossing algorithm, described in section "Modification of zero crossing method: improvement of estimation accuracy," and algorithm with harmonic regressor, described in section "Description of the minimal residual method" for the case of frequency tracking, is presented in Figure 6, which shows histograms of the tracking errors; in Figure 7, which shows a time chart of frequency estimates; and in Figure 8, which shows frequency estimates in the case of fast frequency transient.

Statistical hypothesis test shows that the classical algorithm does not estimate time-varying frequency statistically correct (see Figure 6). One-sample $t$-test indicates a rejection in the null hypothesis that the mean value of the data set is zero at a sufficiently low significance level, which means that the algorithm 


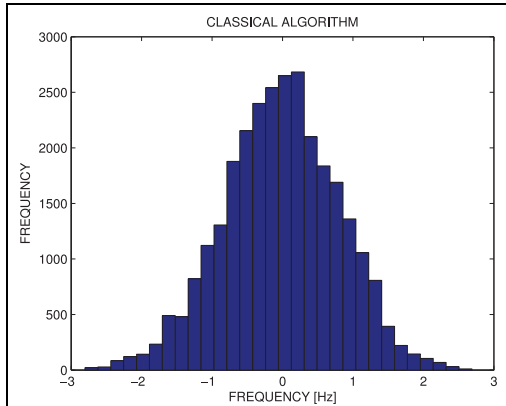

(a)

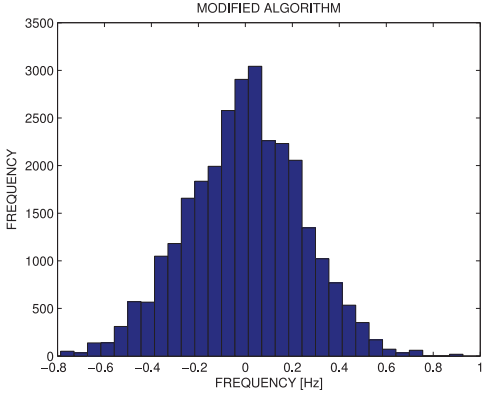

(b)

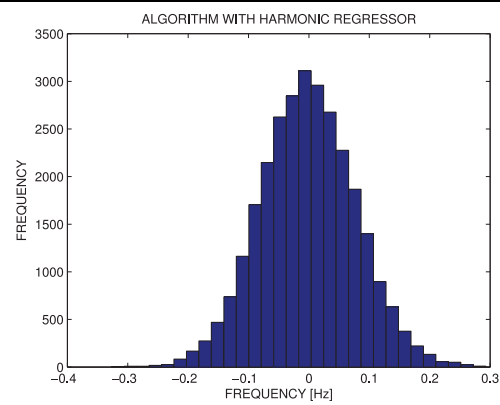

(c)

Figure 6. A signal with time-varying frequency and the second and the fifth harmonic was contaminated with a white noise and processed by classical, and modified zero crossing algorithms, and by the algorithm with harmonic regressor. A time chart of the frequency estimates is shown in Figure 7. Subplots (a), (b) and (c) show histograms of the deviations between actual frequency and estimated frequency for (a) classical zero crossing algorithm, (b) modified zero crossing algorithm and (c) algorithm with harmonic regressor.

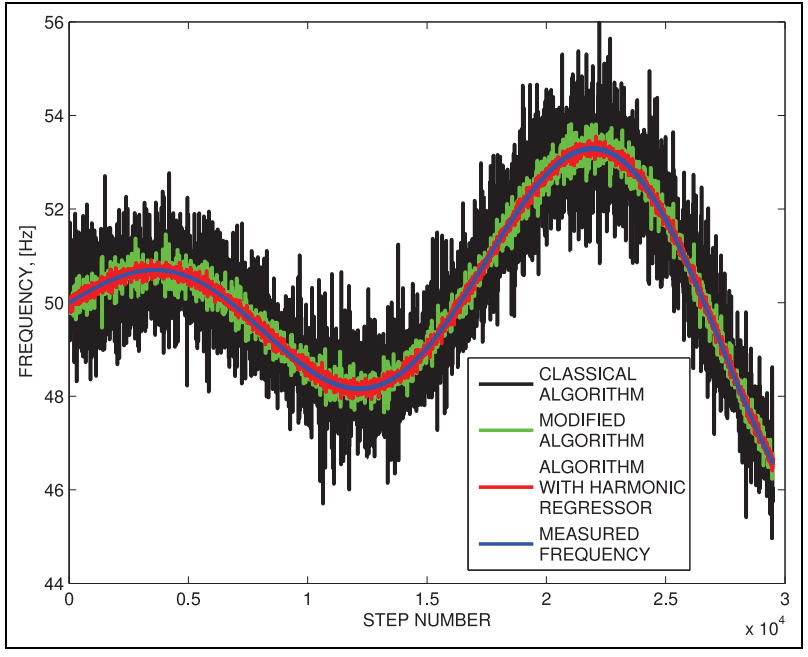

Figure 7. The signal with time-varying frequency and the second and the fifth harmonic was processed for frequency estimation by three algorithms: (I) classical zero crossing algorithm, plotted with a black line; (2) modified algorithm, plotted with a green line; and (3) algorithm with harmonic regressor, plotted with a red line. Actual frequency of the signal is plotted with a blue line. Histograms of the estimation errors are plotted in Figure 6.

provides biased estimation. Modified algorithm and algorithm with harmonic regressor provide statistically correct estimates of time-varying frequency according to the same test. Besides, two-sample $F$-tests for equal variances show that the reduction of the variance provided by the modified algorithm with respect to the classical algorithm and the reduction of the variance provided by the algorithm with harmonic regressor with respect to the modified algorithm are statistically significant. It means that modified zero crossing method provides significant improvement of estimation performance compared to the classical method, and the algorithm with harmonic regressor provides further significant improvement compared to modified zero crossing method.

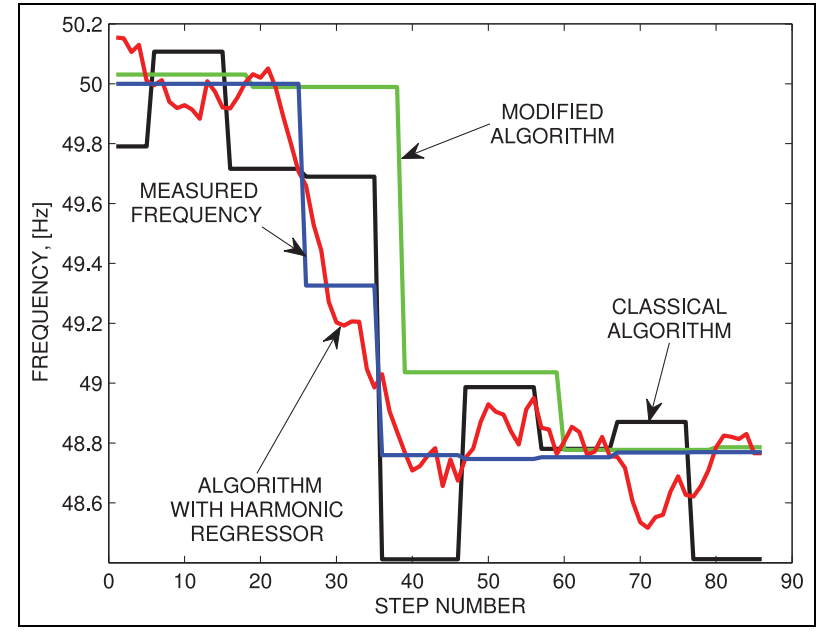

Figure 8. The signal of $50 \mathrm{~Hz}$ in the case of significant load event in electricity network was processed for frequency tracking by three algorithms: (I) classical zero crossing algorithm, plotted with a black line; (2) modified algorithm, plotted with a green line; and (3) algorithm with harmonic regressor, plotted with a red line. Actual frequency is plotted with a blue line.

Notice that the same conclusions are valid in the case of frequency determination (where actual frequency is constant) for which the histograms are similar to histograms presented in Figure 6.

Figure 8, which shows the frequency estimates in the case of significant load event in electricity network, indicates a significant delay in the estimate, provided by the modified algorithm. Algorithm with harmonic regressor provides the best estimate, compared to two other algorithms in the case of fast frequency transient.

Besides, algorithm with harmonic regressor provides frequency estimate in each step of moving window, whereas classical and modified algorithms update the estimates at each zero crossing event only. Therefore, the quality of the derivative signal of the frequency, ${ }^{21}$ which is very important in the frequency control 
problems, is better for the algorithm with harmonic regressor. This in turn has a significant impact on the performance of frequency regulation and in turn on the safety, stability and efficiency of overall electricity network.

\section{Conclusion and discussion: the transition from zero crossing method to complete reconstruction of the frequency contents of the signals}

Significant distortions associated with harmonics and fast changes of the fundamental frequency of voltage and current signals are expected in future electricity networks. These disturbances will also appear around zero crossing points of the signals, deteriorating accuracy of the grid frequency estimation via classical zero crossing method. Modifications of zero crossing method, aiming for improvement of estimation accuracy, are all based on more accurate detection of zero crossing point and require additional signal processing techniques, such as least-squares method, described in section "Modification of zero crossing method: improvement of estimation accuracy." Least-squares method requires in turn some delay for the best fit. This delay is significant for noise-contaminated signals with a large number of harmonics. This delay introduces significant limitations in the performance of modified zero crossing methods in the case of fast frequency tracking, see, for example, Figure 8 for details. In other words, such limitations are associated with a trade-off between the quality of the frequency estimation signal for constant (or slowly varying) frequency and tracking performance in the case of fast changes of the frequency.

Taking into account such limitations, future frequency estimation algorithms should be model based, which allows complete reconstruction of the frequency contents of the signals (over a moving window with a changeable size), and recovering fast frequency variations from noise-contaminated signals. Such algorithms, which are based on multiple models and require minimum information about the frequency content of signal, are described in section "Systems with harmonic regressor: multiple model approach." Simulation results show statistically significant improvement of estimation performance compared to classical zero crossing method and its modifications for frequency tracking.

Finally, future parallel computing technologies, which allow simultaneous calculations for multiple models, will result in fast and high-performance frequency estimation.

\section{Declaration of conflicting interests}

The author(s) declared no potential conflicts of interest with respect to the research, authorship and/or publication of this article.

\section{Funding}

The author(s) received no financial support for the research, authorship and/or publication of this article.

\section{References}

1. Friedman V. A zero crossing algorithm for the estimation of the frequency of a single sinusoid in white noise. IEEE T Signal Proces 1994; 42(6): 1565-1569.

2. Gupta A, Thakur R and Murarka S. An efficient approach to zero crossing detection based on opto-coupler. Int J Eng Res Appl 2013; 3(5): 834-838.

3. Köhler B, Hennig C and Orglmeister R. QRS detection using zero crossing counts. Prog Biomed Res 2003; 8(3): $138-145$.

4. Grillo D, Pasquino N, Angrisani L, et al. An efficient extension of the zero-crossing technique to measure frequency of noisy signals. In: Proceedings of the IEEE international instrumentation and measurement technology conference (I2MTC), Graz, Austria, 13-16 May 2012. New York: IEEE.

5. Wall R. Simple methods for detecting zero crossing. In: Proceedings of the 29th annual conference of the IEEE industrial electronics society (IECON '03), Roanoke, VA, 2-6 November 2003, pp.10-30. New York: IEEE.

6. Duric M and Durisic R. Combined Fourier and zero crossing technique for frequency measurement in power networks in the presence of harmonics. $R E \& P Q J$ 2010; 1(3): 463-469.

7. Jacobsen E and Kootsookos P. Fast, accurate frequency estimators. IEEE Signal Proc Mag 2007; 24(3): 123-125.

8. Kim Y, Kim C, Ban W, et al. A comparative study on frequency estimation methods. J Electr Eng Technol 2013; 8(1): 70-79.

9. Quinn B and Hannan E. The estimation and tracking of frequency, part of Cambridge series in statistical and probabilistic mathematics. Cambridge: Cambridge University Press, 2013.

10. Bobtsov A, Efimov D, Pyrkin A, et al. Switched algorithm for frequency estimation with noise rejection. IEEE T Automat Contr 2012; 57(9): 2400-2404.

11. Pin G, Chen B, Parisini T, et al. Robust sinusoid identification with structured and unstructured measurement uncertainties. IEEE T Automat Contr 2014; 59(6): 1588-1593.

12. Belleter D, Galeazzi R and Fossen T. Experimental verification of a global exponential stable nonlinear wave encounter frequency estimator. Ocean Eng 2015; 97: 48-56.

13. Xia X. Global frequency estimation using adaptive identifiers. IEEE T Automat Contr 2002; 47(7): 1188-1193.

14. Stotsky A. Frequency determination in control applications: excitation-based approach. Proc IMechE, Part I: $J$ Systems and Control Engineering 2012; 226(8): 1142-1148.

15. Walker A. On the estimation of a harmonic component in a time series with stationary independent residuals. Biometrika 1971; 58(1): 21-36.

16. Li T-H. A fast algorithm for efficient estimation of frequencies. In: Proceedings of the signal processing IX: theories and applications: proceedings of EUSIPCO-98 (eds S Theodoridis, I Pitas, A Stouraitis and N Kalouptsidis), Island of Rhodes, Greece: University of Groningen, Johann Bernoulli Institute for Mathematics and Computer Science, 8-10 August 1998, vol. 1, pp.65-68. 
17. Stotsky A. Automotive engines: control, estimation, statistical detection. Berlin, Heidelberg: Springer-Verlag, 2009.

18. Stotsky A. Combined high-order algorithms in robust least-squares estimation with harmonic regressor and strictly diagonally dominant information matrix. Proc IMechE, Part I: J Systems and Control Engineering 2015; 229(2): 184-190.

19. Stotsky A. Accuracy improvement in least-squares estimation with harmonic regressor: new preconditioning and correction methods. In: Proceedings of the IEEE 54th annual conference on decision and control, Osaka, Japan, 15-18 December 2015, pp.4035-4040. New York: IEEE.

20. Amdahl G. Validity of the single processor approach to achieving large-scale computing capabilities. AFIPS Conf $P$ 1967; 30: 483-485.

21. Stotsky A and Forgo A. Recursive spline interpolation method for real-time engine control applications. Control Eng Pract 2004; 12: 409-416.

22. Benzi M. Preconditioning techniques for large linear systems: a survey. J Comput Phys 2002; 182: 418-477.

23. Chen K. Matrix preconditioning techniques and applications. Cambridge: Cambridge University Press, 2005.

24. Horn R and Johnson C. Matrix analysis. Cambridge: Cambridge University Press, 1985.

25. Durand E. Solutions numeriques des equations algebriques, vol. 2. Paris: Masson, 1972.

26. Gustafsson F. Adaptive filtering and change detection. Hoboken, NJ: John Wiley \& Sons, 2000.

\section{Appendix I}

\section{New family of high-order algorithms and stepwise splitting method}

\section{High-order algorithm description: additive splitting, matrix inversion lemma and preconditioning}

Any positive definite and symmetric matrix $A$ can be split as follows (see, for example, Benzi, ${ }^{22} \mathrm{Chen}^{23}$ and references therein)

$$
A=S-D
$$

where $S$ is a positive definite and symmetric matrix, and $D$ is a symmetric matrix. Possible choices of matrices $S$ and $D$ for an additive splitting of $A$ are discussed below.

Splitting (14) facilitates calculation of the inverse of matrix $A$, which can be used as a preconditioner for high-order algorithms ${ }^{18,19}$ that solve the equation $A \theta=b$ with respect to the parameter vector $\theta$, see section "Description of the minimal residual method."

New family of algorithms of order $h=1,2, \ldots$ can be obtained via sequential application of the matrix inversion lemma (Woodbury matrix identity)

$$
\begin{aligned}
& G_{k}=\left(S^{-1} D\right)^{h} G_{k-1}\left(D S^{-1}\right)^{h} \\
& +\sum_{j=0}^{h-1}\left(S^{-1} D\right)^{j}\left[S^{-1}+S^{-1} D S^{-1}\right]\left(D S^{-1}\right)^{j}
\end{aligned}
$$

where $G_{k}$ is an estimate of $A^{-1}, G_{0}$ is arbitrary $\left(G_{0}\right.$ can be chosen as an inverse diagonal matrix of the diagonal entries of $A$ ), the spectral radius $\rho\left(S^{-1} D\right)<1$, $k=1,2, \ldots$ The matrix $S$ is easy invertible matrix (or the matrix whose inverse is known). For example, for an SDD matrix $S$, the inverse can be calculated easily and accurately using high-order algorithms described in Stotsky. ${ }^{18}$ In addition, the inverse of $S$ is known in the recursive calculations of the inverse of information matrix in a moving window, see section "Application to recursive calculation of the inverse of information matrix in moving window." Splitting (14) can also be written in the following form

$$
I-S^{-1} A=S^{-1} D
$$

where $I$ is the identity matrix, and $\rho\left(S^{-1} D\right)<1$ for symmetric and positive definite matrices $A$ and $S$, provided that $2 S-A$ is a positive definite matrix, which imposes restriction on the choice of $S$ and $D .^{24}$

Notice that the matrix $S^{-1}$ can be used as a preconditioner for high-order algorithms described in Stotsky ${ }^{18,19}$ provided that $2 S>A$.

Comparison of high-order algorithm (15) and highorder algorithms described in Stotsky ${ }^{18,19}$ is given in section "Comparison."

The family of high-order algorithms (15) is presented in Table 1.

Algorithm (15) has the following error model

$$
G_{k}-A^{-1}=\left(S^{-1} D\right)^{h}\left\{G_{k-1}-A^{-1}\right\}\left(D S^{-1}\right)^{h}
$$

which can be written in the following form

$$
G_{k}-A^{-1}=\left(S^{-1} D\right)^{h k}\left\{G_{0}-A^{-1}\right\}\left(D S^{-1}\right)^{h k}
$$

The convergence rate increases with the order $h$ and is determined by the eigenvalues of the matrix $S^{-1} D$, which are the same as eigenvalues of $D S^{-1}$.

Notice the following simplified version

$$
G_{k}=\left(S^{-1} D\right) G_{k-1}+S^{-1}
$$

of the first-order algorithm in Table 1 is presented in Durand. $^{25}$ The convergence rate of the first-order algorithm in Table 1 is at least two times higher than the convergence rate of truncated algorithm (19).

Splitting (14) provides the basis for a new method, which is called stepwise splitting, described in section "Stepwise splitting and matrix inversion by parts using high-order algorithms," and gives a flexible framework for calculation of preconditioners for different types of matrices, see sections "Splitting for positive definite, symmetric and almost SDD matrix A" and section "Splitting for an SDD matrix A." It is shown that stepwise splitting can be applied to recursive calculation of the inverse of information matrix in moving window, see section "Application to recursive calculation of the inverse of information matrix in moving window," and 
Table I. New family of high-order matrix inversion algorithms.

\begin{tabular}{ll}
\hline Order & $\begin{array}{l}\text { Matrix inversion } \\
\text { algorithms, } G_{k}=\end{array}$ \\
\hline 1 & $\left(S^{-1} D\right) G_{k-1}\left(D S^{-1}\right)+\left[S^{-1}+S^{-1} D S^{-1}\right]$ \\
2 & $\left(S^{-1} D\right)^{2} G_{k-1}\left(D S^{-1}\right)^{2}+\sum_{j=0}^{1}\left(S^{-1} D\right)^{j}\left[S^{-1}+S^{-1} D S^{-1}\right]\left(D S^{-1}\right)^{j}$ \\
3 & $\left(S^{-1} D\right)^{3} G_{k-1}\left(D S^{-1}\right)^{3}+\sum_{j=0}^{2}\left(S^{-1} D\right)^{j}\left[S^{-1}+S^{-1} D S^{-1}\right]\left(D S^{-1}\right)^{j}$ \\
$\ldots$ & $\ldots$ \\
$h$ & $\left(S^{-1} D\right)^{h} G_{k-1}\left(D S^{-1}\right)^{h}+\sum_{j=0}^{h-1}\left(S^{-1} D\right)^{j}\left[S^{-1}+S^{-1} D S^{-1}\right]\left(D S^{-1}\right)^{j}$, \\
& $\rho\left(S^{-1} D\right)<1$
\end{tabular}

to inversion of ill-conditioned matrices, see section "Application to positive definite, symmetric and illconditioned matrices."

\section{Stepwise splitting and matrix inversion by parts using high-order algorithms}

Suppose that the matrix $A$ is presented in the form (14), where $D$ and $S$ are symmetric matrices (The stepwise splitting method is also applicable for the nonpositive definite matrices $S$. Notice that the information matrix $S$ is the positive definite matrix for systems with harmonic regressor, see, for example, the application to recursive calculations described in section "Application to recursive calculation of the inverse of information matrix in moving window") and $S^{-1}$ is known. Suppose that $\rho\left(S^{-1} D\right) \geqslant 1$, and high-order algorithms cannot be applied directly for inversion of the matrix $A$. Nevertheless, the inverse of this matrix can be calculated by parts with the stepwise splitting algorithm described below.

This algorithm represents the calculations of the finite sequence of matrices $S_{i}$ and $D_{i}$ (initialized as $S_{1}=S$ and $D_{1}=D$ in (14)) and the associated sequence of scaling factors $\alpha_{i}$, where $i=1,2, \ldots, i_{*}$, and $\alpha_{i *}=1$ such that

$$
\begin{aligned}
& \alpha_{i}=\left\|S_{i}^{-1} D_{i}\right\|_{\infty}+\varepsilon \\
& A=\underbrace{\left\{S_{i}-\frac{1}{\alpha_{i}} D_{i}\right\}}_{S_{i+1}}-\underbrace{\frac{\alpha_{i}-1}{\alpha_{i}} D_{i}}_{D_{i+1}}
\end{aligned}
$$

where $S_{i}-\left(1 / \alpha_{i}\right) D_{i}$, and $\left(\left(\alpha_{i}-1\right) / \alpha_{i}\right) D_{i}$ are denoted as $S_{i+1}$ and $D_{i+1}$, respectively, in each step, and $i=i+1$ while $\alpha_{i}>1$, and $\varepsilon$ is a small positive number. Algorithm converges when $\alpha_{i}$, calculated in (20) is less than one (or equal to one) and is assigned to one $\alpha_{i *}=1$. The norm $\|\cdot\|_{\infty}$ is defined as the maximum absolute row sum norm.

The inverse of the matrix $S_{i+1}=S_{i}-\left(1 / \alpha_{i}\right) D_{i}$, which is presented in the form (14), is calculated using high-order algorithm (15) or algorithm described in Stotsky ${ }^{18}$ with preconditioning matrix $S_{i}^{-1}$. The choice of $\alpha_{i}$ in (20) guarantees that the following norm is less than one in each step, that is

$$
\left\|I-S_{i}^{-1}\left(S_{i}-\frac{D_{i}}{\alpha_{i}}\right)\right\|_{\infty}=\frac{\left\|S_{i}^{-1} D_{i}\right\|_{\infty}}{\alpha_{i}}<1
$$

and minimizes the number of steps of the algorithm. The matrix $A$ remains the same in each step, and the matrix $D_{i}$ is stepwise scaled so that the convergence condition (22) is satisfied.

Stepwise splitting procedure associated with (20) and (21) is the transformation of splitting (14) to the following splitting

$$
\begin{aligned}
& A=S_{i *}-D_{i *} \\
& \left\|I-S_{i *}^{-1} A\right\|_{\infty}=\left\|S_{i *}^{-1} D_{i *}\right\|_{\infty}<1
\end{aligned}
$$

where $\alpha_{i *}=1$. Any high-order algorithm can be applied for accurate calculation of the inverse of matrix $A$ (and the parameter vector) with $S_{i *}^{-1}$ as a preconditioning matrix.

The algorithm provides calculation of the inverse of matrix $A$ with improved accuracy compared to the calculation based on straightforward application of the matrix inversion lemma without accuracy control.

\section{Application to recursive calculation of the inverse of information} matrix in moving window. Consider evolution of information matrix $A$ in one step of the window which is moving in time, represented in the form (14), where $A$ is an information matrix in the current step, $S$ is information matrix in the previous step and finally $D$ is the difference between two rank one matrices, associated with regressors which enter and leave the window, see Stotsky $^{17}$ and Gustafsson ${ }^{26}$ for details. Notice that the matrix $D$ may even contain the sum of a number of rank one matrices, if the window changes its size in the case of transient, for example. Notice that the inverse of the matrix $S$ is known from the previous step. The inverse of the information matrix is calculated in each step using information about the inverse of the matrix $S$ from the previous step and stepwise splitting algorithm (20 and 21). The number of steps of the algorithm depends on the window size and decreases, if the window size increases.

Application to positive definite, symmetric and ill-conditioned matrices. Stepwise splitting method can also be applied to ill-conditioned matrices for which the condition number is too large. Ill-conditioned matrix $A$ can always be presented in the form (14), where $S$ is an SDD positive definite matrix and $D$ is the diagonal matrix. An SDD matrix $S$ is easy invertible matrix and small elements are added stepwise to the diagonal elements of $S$ for accurate calculations of inverse of illconditioned matrix, using algorithm (20 and 21).

Reduced orders and number of steps can be used in matrix inversion algorithm (15) in the stepwise method compared to the method described in Stotsky ${ }^{19}$ for illconditioned matrices. Such reduction makes stepwise method more computationally efficient compared to other methods. 
Notice that the algorithm is also very efficient for calculation of approximate inverse of rank-deficient matrices.

\section{Splitting for positive definite, symmetric and almost SDD matrix $A$}

The matrix $S$ can be chosen as an SDD matrix in (14) and $D$ can be chosen as a diagonal matrix as it is described in section "Application to positive definite, symmetric and ill-conditioned matrices." Diagonal elements of $D$ are the complements/corrections to the diagonal elements of $A$ in this case so that the matrix $S=A+D$ is an SDD matrix.

The elements of the matrix $D$ can be chosen as follows. If a diagonal element of the matrix $A$ in a row is larger than the sum of absolute values of other elements in that row, then corresponding element in the matrix $D$ is equal to zero. In the rows where diagonal elements of the matrix $A$ are less than the sums of absolute values of other elements the minimal positive values should be chosen as diagonal elements of the matrix $D$ (providing row wise corrections), so that the matrix $S=A+D$ becomes an SDD matrix. Such a presentation can be applied to the matrices for which diagonal elements dominate in a number of rows and/ or diagonal elements in the rows are slightly less than the sums of absolute values of other elements in these rows. In other words, the matrix $A$ is almost SDD matrix (close to SDD matrix). The matrix $D$ contains zero and small positive diagonal elements only in this case, which ensures that the spectral radius is less than one, $\rho\left(S^{-1} D\right)<1$.

\section{Splitting for an SDD matrix A}

In this case, the matrix $S$ can be chosen as a diagonal matrix in (14), which contains the diagonal elements of an SDD matrix $A$, and the matrix $D$ with zero diagonal elements contains off-diagonal elements of the matrix $A$. Then, $\left\|S^{-1} D\right\|_{\infty}<1$, see (16).

Notice that other types of splitting are also described in Benzi ${ }^{22}$ and $\mathrm{Chen}^{23}$ (see also references therein).

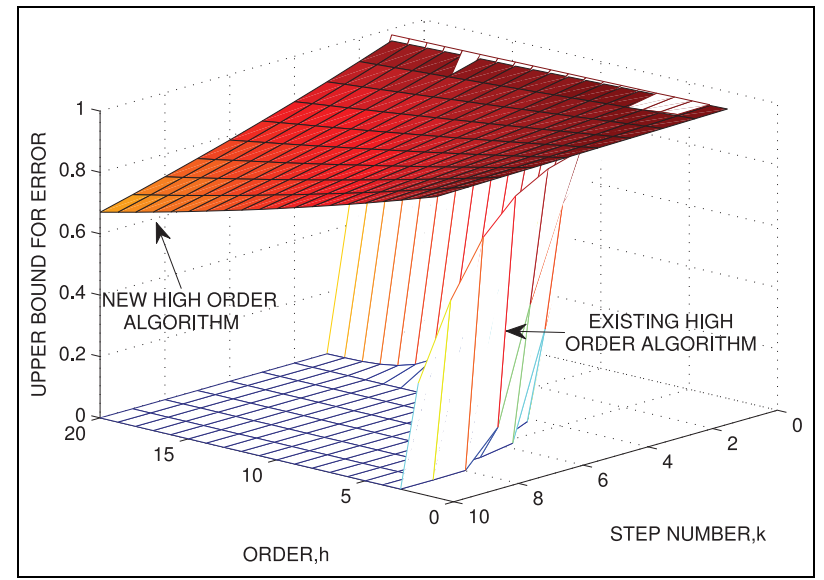

Figure 9. Two transient upper bounds of the matrix inversion errors defined as $\kappa^{2 h k}$ with $h=1,2, \ldots, 20$ for algorithm (I5) (named as "new high-order algorithm"), and defined as $\kappa^{h^{k}}$ with $h=2,3, \ldots, 20$ for the algorithm in Stotsky ${ }^{19}$ (named as "existing high-order algorithm") are plotted as red and white surfaces, respectively, where $\kappa=0.999$, and $h$ is the order of the algorithms, $k=1,2, \ldots, 10$.

\section{Comparison}

Performance of the algorithm (15) can be compared to the performance of the high-order matrix inversion algorithm described in Stotsky ${ }^{19}$ for inversion of positive definite symmetric matrices. Information matrix associated with the system with harmonic regressor with three frequencies is used for comparison.

Transient upper bound of the estimation error is defined as $\kappa^{2 h k}$ for algorithm (15), and the same bound is defined by $\kappa^{h^{k}}$ for the algorithm in Stotsky,${ }^{19}$ where $h$ is the order of the algorithms, $k=1,2, \ldots$ The number $0<\kappa<1$ is associated with the spectral radius of the matrix $S^{-1} D$. Comparison of two upper bounds associated with algorithm (15) and algorithm described in Stotsky ${ }^{19}$ is presented in Figure 9. High-order algorithm described in Stotsky ${ }^{19}$ has significantly higher convergence rate, excepting for the case of low orders, where the rates are comparable in the initial steps of estimation.

Notice that the transient upper bounds on estimation errors do not give complete picture of the performance of the algorithms. 